\title{
Mode-Locking Dynamics in a Quantum-Dash Fabry-Pérot Laser Diode for Packet Based Clock Recovery Applications
}

\author{
Ramón Maldonado-Basilio, ${ }^{1}$ Josué Parra-Cetina, ${ }^{1}$ Sylwester Latkowski, ${ }^{1}$ Pascal Landais, ${ }^{1}$ Nicola Calabretta ${ }^{2}$ \\ ${ }^{I}$ The RINCE Institute, School of Electronic Engineering, Dublin City University. Collins Avenue Extension, Glasnevin, Dublin 9, Ireland \\ ${ }^{2}$ Eindhoven University of Technology, dept. Electrical Engineering, 5600MB Eindhoven, The Netherland \\ Corresponding author: Ramon.Maldonado@dcu.ie
}

\begin{abstract}
We experimentally investigate the locking/unlocking dynamics of a mode-locked QDash laser diode for packet-based clock-recovery applications. Results show 20 ns locking times for the passively and externally synchronized mode-locking mechanisms.
\end{abstract}

\section{Introduction}

The exponential growth of traffic in access networks makes it likely that future optical networks should be capable of handling tens of $\mathrm{Tb} / \mathrm{s}$ data traffic. To cope with this data traffic, links interconnecting the nodes of the network will carry optical packets at data rates above $100 \mathrm{~Gb} / \mathrm{s}$. Assuming $100 \mathrm{~Gb} / \mathrm{s}$ data packets with typically 1500 bytes, the length of each packet would be around $120 \mathrm{~ns}$. To optimize the load at which such a system can operate, the guard times between the packets have to be small, in the order of a few tens of nanoseconds. This requires that the system has a receiver with a packet based clock extraction circuit with very fast locking/unlocking times (few tens of ns) to implement a data burst reception at high bit rate. Clock recovery at high data rate is typically performed by using a phase-locked loop circuitry for synchronizing the local clock with the data signal. In this technique, the synchronizing (locking) time works inversely with the timing jitter: shorter locking time means larger timing jitter. At data rates above $160 \mathrm{~Gb} / \mathrm{s}$, the very high time resolution required for the short duration of the bit time slot will result in a locking range in the order of microseconds. This prevents the application of this technique for clock recovery of data packets which have a typical duration of a few hundred nanoseconds. While several compact semiconductor devices have been employed for clock recovery at bit rate beyond $160 \mathrm{~Gb} / \mathrm{s}$ [1,2], packet based clock extraction with a very fast locking/unlocking times has only been demonstrated up to $40 \mathrm{~Gb} / \mathrm{s}$ by using several Mach-Zehnder interferometers along with semiconductor optical amplifiers [3]. In this work we present, for the first time to the author's knowledge, an experimental study of the switching and locking times of the mode-locking mechanism in a quantum-dash (QDash) Fabry-Pérot laser diode. The proposed approach is based on an analysis of the instantaneous frequency of the $\sim 40 \mathrm{GHz}$ beat-tone signal measured at the output of the laser under investigation, which after a frequency down-conversion stage, is recorded by a real-time oscilloscope (RTO). Experimental results show that the QDash laser diode is passively mode-locked in less than $50 \mathrm{~ns}$ after switching on the device and instantaneously unlocked when the laser is switched off. These results indicate that the QDash PML-LD under investigation is a promising compact device for realizing a packet based clock recovery.

\section{Experiment}

The device under investigation is a $\sim 1 \mathrm{~mm}$ long, unpackaged single section QDash Fabry-Pérot laser diode, without a saturable absorber section [4]. This passively mode-locked laser diode (PML-LD) presents a bias threshold of $18 \mathrm{~mA}$ at $25^{\circ} \mathrm{C}$, and provides an average optical power of $0.4 \mathrm{~mW}$ at $100 \mathrm{~mA}$. It features more than 40 longitudinal modes with $0.31 \mathrm{~nm}$ inter-modal separation, resulting in a $3 \mathrm{~dB}$ optical bandwidth of $12 \mathrm{~nm}$ centered at $1526 \mathrm{~nm}$. Experimental setup to assess the passive locking/unlocking time when the PML-LD is switching from the off to on state is depicted in Fig. 1. Bias current is provided by a synthesized clock generator set at $40 \mathrm{kHz}$ and duty cycle at $50 \%$, with low and high current levels set at 0 and $100 \mathrm{~mA}$, respectively. The selected frequency of the clock generator allows an efficient use of the RTO in terms of its internal buffer size and sampling rate, whereas it is sufficient to observe the dynamic behavior of the tested device. Moreover, the selection of the bias current upper level relies on the operating conditions of the PML-LD at which it synchronizes to an externally injected signal [5]. The optical power emitted from the laser is collected through a lensed fibre and the $\sim 40 \mathrm{GHz}$ beat-tone signal is detected on a fast $(50 \mathrm{GHz})$ photo-detector (PD) followed by an RF-amplifier. Taking into account the features of the RTO used in this experiment (10 GHz frequency bandwidth and $40 \mathrm{GS} / \mathrm{s}$ sampling rate), the beat-tone signal of interest is frequency down-converted (FDC) to $\sim 1 \mathrm{GHz}$ by using an RF-mixer and a local oscillator (LO) at $\sim 39 \mathrm{GHz}$. The signal from the $\mathrm{LO}$ features a linewidth of a few $\mathrm{Hz}$, affecting to a minimal extent the characteristics 

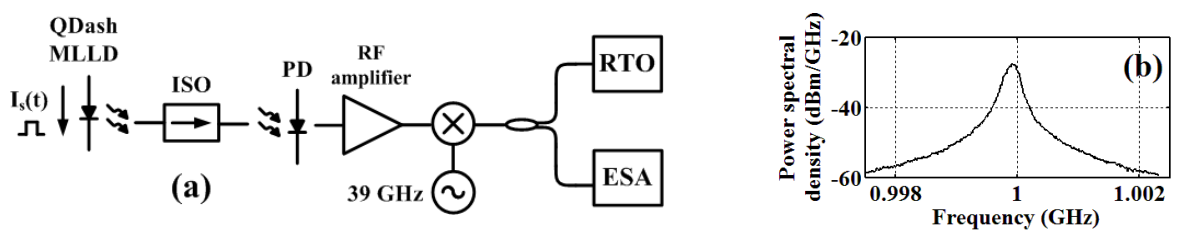

Fig. 1. (a) Experimental setup for assessing the switching time of the passive mode-locking mechanism. (b) Frequency down-converted beat-tone measured at the output of the RF-mixer.

of the $\sim 40 \mathrm{GHz}$ beat-tone after the RF-mixer. A trace of the $\sim 1 \mathrm{GHz}$ FDC beat-tone signal measured at the mixer output with an electrical spectrum analyzer (ESA) is depicted in Fig. 1(b) whilst the PML-LD is DC-biased at $100 \mathrm{~mA}$.

\section{Results}

First we study the free-running (no external optical injection) operation of the PML-LD when a constant bias current of $100 \mathrm{~mA}$ is supplied to the laser. Fig. 2(a) shows the FDC beat-tone measured with the RTO. A stable frequency component of $\sim 1 \mathrm{GHz}$ is clearly observed in this signal, as depicted in the zoom-in trace of Fig. 2(b). Then the PML-LD is operated with a $40 \mathrm{kHz}$ modulated bias current as shown in Fig. 2(c). From the zoom-in plot depicted in Fig. 2(d), a rise-time (from $10 \%$ to $90 \%$ ) of $\sim 8 \mathrm{~ns}$ is measured on the biasing current function. The time domain trace of the FDC beat-tone is shown in Fig. 2(e). Analyzing the emitted power of the QDash PML-LD (visualized in these plots as the envelope of the beat-tone), it does not exhibit an instantaneous response with the current supplied, particularly after the threshold current $\left(\mathrm{I}_{\mathrm{th}}\right)$ has been reached. A switch on time $\tau_{0}$ of around 30 ns is observed, as revealed in the zoom-in trace shown in Fig. 2(f). Furthermore, analyzing the frequency of the down-converted beattones, it stabilizes to a constant value of $\sim 1 \mathrm{GHz}$ only after a response time $\tau_{1}$ of $\sim 50 \mathrm{~ns}$. This means that the passive mode-locking process taking place in the QDash PML-LD occurs $\sim 20 \mathrm{~ns}$ after the switch on time. An immediate response of the passive mode-locking process is observed after switching off the bias current. The instantaneous frequency exhibited by the recorded FDC beat-tones is shown in Figs. 2(g) and 2(h). The red-dashed and blue traces represent the frequency of the beat-tone when the PML-LD is biased with a DC current of $100 \mathrm{~mA}$ and with the $40 \mathrm{kHz}$ modulated current, respectively. It is worth mentioning that the instantaneous frequency of the down-
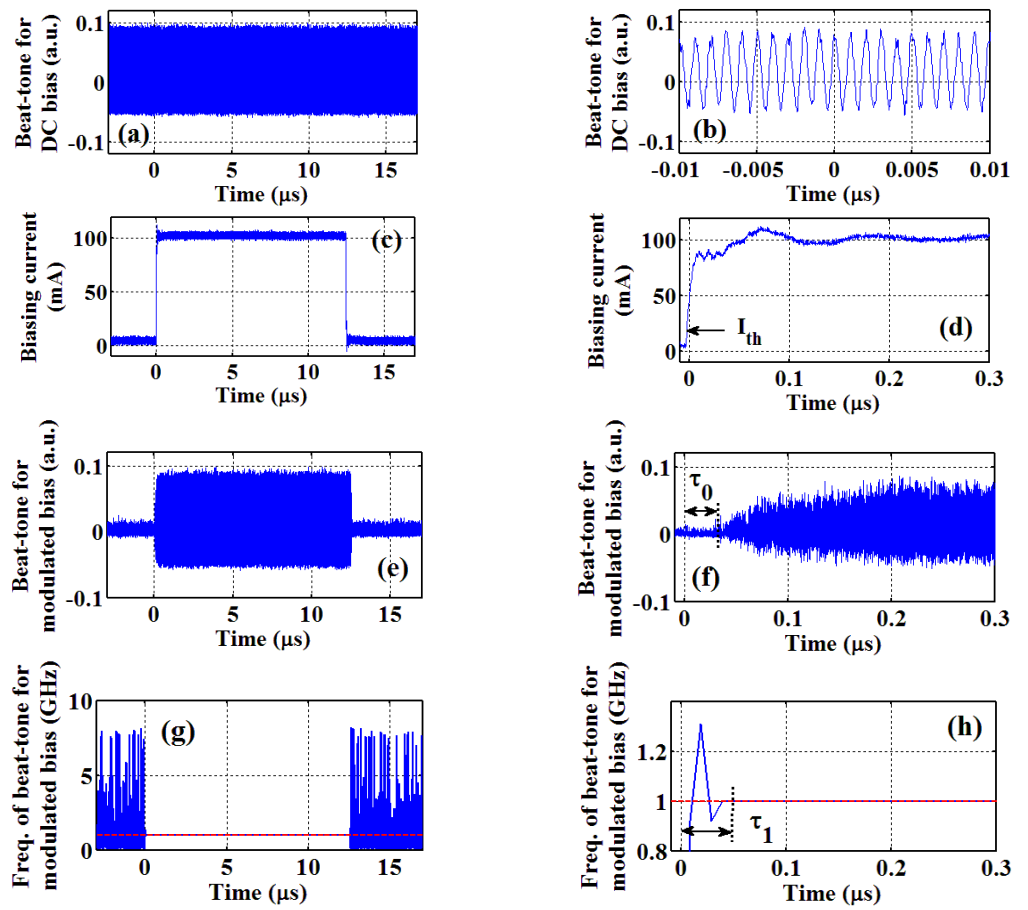

Fig. 2. (a)-(b) Frequency down-converted beat-tone in constant current conditions. (c)-(d) Time-domain traces of the modulating bias current source. (e)-(f) Frequency down-converted beat-tone after applying the modulating bias current. (g)-(h) Instantaneous frequency of the recorded beat-tone both in constant current (red) and after current modulation (blue). 
converted beat-tones has been obtained by implementing a fast-Fourier-transform (FFT) to the recorded data in time-windows of $10 \mathrm{~ns}$. The selected window determines the accuracy of the retrieved switching time $\tau_{1}$, and also sets a resolution of $\sim 80 \mathrm{MHz}$ for the instantaneous frequency of the down-converted beat-tone.

Experiments to assess the locking/unlocking times when the PML-LD is synchronized (and desynchronized) to the injection of an external train of $10 \mathrm{GHz}$ optical pulses are also implemented. The experimental setup is illustrated in Fig. 3(a). A train of $10 \mathrm{GHz}$ optical pulses with a pulse width of $\sim 1.6$ ps generated by a tunable modelocked laser (TMLL) is amplitude modulated by the synthesized clock generator, set again at $40 \mathrm{kHz}$ and a duty cycle at $50 \%$. This optical signal with such a duty cycle allows for investigation of the periodical synchronization (and desynchronization) of the QDash PML-LD with a period of $12.5 \mu \mathrm{s}$. The laser is biased at a constant current of $100 \mathrm{~mA}$. The optical power of the $10 \mathrm{GHz}$ pulse train required to achieve the external synchronization ranges from 9 to $11 \mathrm{dBm}$ (measured at the input of the lensed fiber). The central frequency of the PML-LD when the device operates in free running and under external synchronization are first measured by using an ESA set at a resolution of $300 \mathrm{kHz}$ and a span of $50 \mathrm{MHz}$. As depicted in Fig. 3(b), a shift of $\sim 11 \mathrm{MHz}$ between the beat-tones distinguishes one and the other operating conditions. Following our proposed approach, $20 \mu \mathrm{s} \mathrm{time-window} \mathrm{of} \mathrm{the} \mathrm{FDC} \mathrm{beat-tones}$ are recorded by the RTO when the device operates both in free-running and under synchronization. Implementing an FFT to the FDC beat-tone in free-running, a single component centered at around $1 \mathrm{GHz}$ is obtained, as depicted in Fig. 3(c) in the blue trace. On the other hand, after implementing an FFT to the FDC beat-tone measured under synchronization, a single frequency component is retrieved instead of dual components with $\sim 11 \mathrm{MHz}$ separation, as depicted in the red trace of Fig. 3(c). This means that once the PML-LD is synchronized to the optical pulses, there is not an observable transition from synchronization to free-running for the analyzed time-window of $20 \mu \mathrm{s}$. Indeed, similar single frequency components are obtained for a number of data sets recorded at different instants.

Summarizing, we have investigated and measured the dynamics of the mode-locking mechanisms in a QDash PML laser diode. In free running operation, a fast locking time on the order of $20 \mathrm{~ns}$ has been measured when switching on the PML-LD, and an immediate unlocking time in absence of bias current. On the other hand, although the locking time with an external optical pulse train is expected to be similar to the locking time measured in free running operation (20 ns), the device exhibits a hysteresis behavior with respect to the external locking signal. Once the synchronization is achieved, the PML-LD under investigation holds such a state at least for $12.5 \mu \mathrm{s}$ after the external signal is being switched off. These results indicate that the QDash laser diode, controlled by a simple electronics for switching on and off the laser state, is a promising candidate to implement a packet based clock recovery with fast locking/unlocking times in the order of a few tens of nanosenconds.
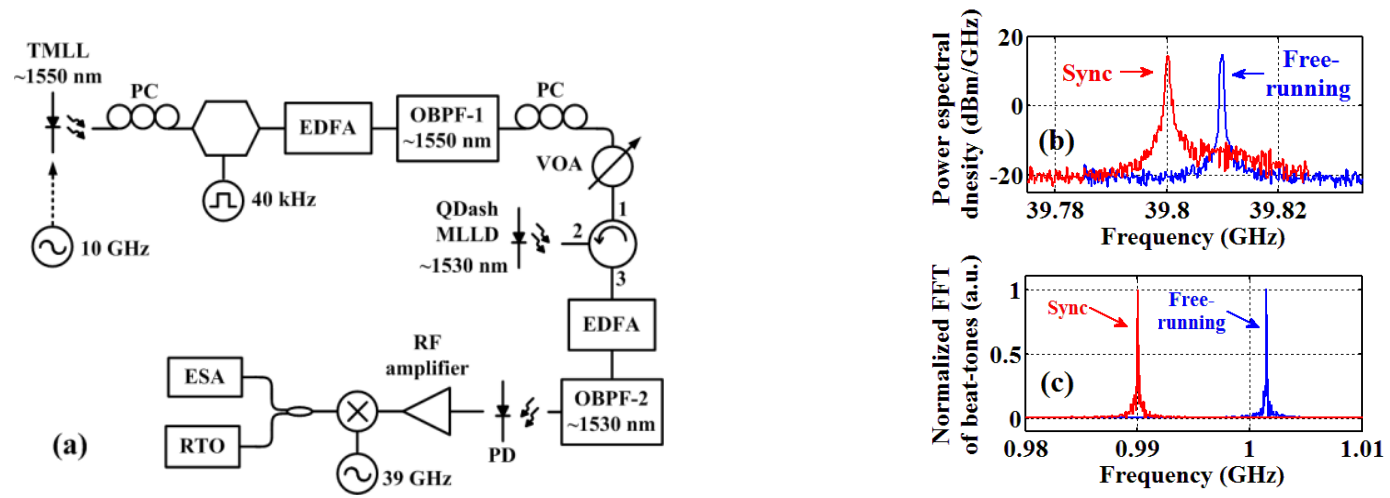

Fig. 3. (a) Experimental setup for assessing the locking time between free-running and external synchronization of the QDash PML-LD under investigation. (b) Beat-tones measured by the ESA and (c) retrieved from the FFT of the recorded frequency down-converted beat-tones .

\section{References}

[1] E. Tangdiongga, H.C. Hansen Mulvad, H. de Waardt, G. D. Khoe, A. M. J. Koonen, and H.J.S. Dorren, "SOA-based Clock Recovery and Demultiplexing in a Lab Trial of 640-Gb/s OTDM Transmission over 50-km Fibre Link," in ECOC, Postdeadline paper PD 1.2 (2007).

[2] V. Roncin, A. O’Hare, S. Lobo, E. Jacquette, L. Bramarie, P. Rochard, Q.-T. Le, M. Gay, J.-C. Simon, A. Shen, J. Renaudier, F. Lelarge, and G.-H. Duan. "Multi-data-rate system performance of a 40-GHz all-optical clock recovery based on a quantum-dot Fabry-Pérot laser." Photonics Technology Letters, 19(19), 1409-1411, (2007).

[3] D. Petrantonakis, G. T. Kanellos, P. Zakynthinos, N. Pleros, D. Apastolopoulos, and H. Avramopoulos. "A 40 Gb/s 3R burst-mode receiver with 4 integrated MZI switches," in Proc. OFC 2006, Paper PDP25 (2006).

[4] F. Lelarge, B. Dagens, J. Renaudier, R. Brenot, A. Accard, F. van Dijk, D. Make, O. Le Gouezigou, J.-G. Provost, F. Poingt, J. Landreau, O. Drisse, El. Derouin, B. Rousseau, F. Pommereau, and G.-H. Duan. "Recent advances on InAs/InP quantum dash based semiconductor lasers and optical amplifiers operating at 1.55 um," IEEE J. Sel. Top. Quantum Electron., 13(1), 111-124 (2007).

[5] R. Maldonado-Basilio, S. Latkowski, S. Philippe, and P. Landais. " $40 \mathrm{GHz}$ mode-beating with $8 \mathrm{~Hz}$ linewidth and 64 fs timing jitter from a synchronized mode-locked quantum-dash laser diode,” Optics Letters, 36(16), 3142-3144 (2011). 